Sir: Dr Al Rubaie's point is well taken. We had certainly not intended to reify the notion of "the unconscious", nor did the therapist or intake team wish to exonerate themselves from clinical responsibility by attributing actions to a notional 'unconscious' rather than to a freely chosen clinical decision.

However, we were trying to draw attention to the fact that clinicians are often unwittingly influenced by forces-perhaps most usefully seen in terms of transference and countertransference - that are outside of their awareness, and that there are interesting overlaps between the field of ethics and such dynamic understanding. So far from absolving ourselves of responsibility by invoking such forces, we suggest that, by becoming aware of them, better clinical and ethical judgements can be made.

JeREMY Holmes, North Devon District Hospital, Barnstaple, Devon EX31 4JB

Sir: Holmes et al, (Psychiatric Bulletin, August $1994,18,466-468$ ) give a valuable demonstration of the utility of philosophy to psychiatry, which they themselves perhaps underestimate.

Justice has indeed been a rather neglected area of psychiatric medical ethics, and its scope extends far beyond resource allocation. For example, Newth (1994) describes the potential for injustice in the treatment of doctors who suffer mental illness. We currently have no way of dealing with this, perhaps because of the countertransference dynamics Holmes et al, describe.

Recent philosophical work may help to resolve one dilemma they posed, that of autonomy and unconscious determinism in psychotherapy. Both psychiatrists and philosophers have usually assumed that our decisions to act are grounded on our desires and our beliefs. This certainly does lead to a problem of competency in psychotherapy, as desires and beliefs in that setting may be determined involuntarlly because of unconscious processes. However, Bratman (1987) has demonstrated that our intentions to act are phenomenologically distinct from both desires and beliefs. He considers intentions are conscious, and involved with planning our decisions. Furthermore, he suggests that they may have a role in preventing incongruent desires and beliefs diverting our plans. They would thus act as a 'filter of admissability' for unconscious desires and motivations. As intentions are conscious and rational, they support autonomous and responsible actions. It may therefore be that our competency in psychotherapy is conferred by our intentions, not our desires or beliefs. Both philosophical and phenomenological research would be needed to test this speculation.
Holmes' et al, discussion of philosophy and ethics can be readily extended to identify a need for new directions in both practice and research. So, philosophical medical ethics can do more than reveal the world as a spectacle, as they claim. It can both study and seek to change it.

Bratman, M. (1987) Intentions, Plans and Practical Reason. Cambridge, Mass: Harvard.

NEWTH, S. (1994) Depressed doctors must 'come out'. BMA News Review, August, 26.

D. M. Foreman, University of Keele, School of Postgraduate Medicine, Stoke-on-Trent ST4 7QB

\section{Group therapy for adults with a learning disability}

Sir: We read with interest the article by $\mathrm{Dr}$ Procter on group therapy with pre-adolescents (Psychiatric Bulletin, August 1994, 18, 485-486) and endorse the value of running groups under supervision for psychiatric trainees.

We recently co-facilitated a seven week group for adults with a learning disability using active techniques under the guidance of a qualified psychodrama therapist. We wanted to provide an opportunity for adults who were in the process of broadening their horizons to meet as a group and share experiences. It had become clear that there were few opportunities for such people to meet new faces outside of the work or home environment. Our leaning towards active techniques came in part from a wish to make the sessions enjoyable for the group members as well as from our own personal and training experiences.

Our aim was for group members to be able to identify feelings within themselves brought up by being in the group as well as to recognise feelings existing within others. In addition we hoped that regular attendance would increase each individual's confidence and level of social skills. Each week one of us started the session with a warm-up exercise and then handed over to the other to complete the main plece of work and end the session. We discussed themes for each session in our weekly supervision but tried to be flexible and change our plans as the need arose.

At the end of the group we felt that we had achieved our aims and the positive response from the members has encouraged us to consider using a similar approach in the future. We learnt a lot both about the practical aspects of setting up a group and therapeutic factors within one. We were also left with much food for thought about the effect that an individual's developmental level may have on his capacity to understand the emotions of another and the 